\title{
Measurement of small rotation angle of flange joints by a novel flexure magnifying mechanism
}

\author{
Xiaotao Zheng ${ }^{1}$, Fen Wei ${ }^{1}$, Haofeng Chen ${ }^{2,3 *}$, Sujuan Guo ${ }^{3}$, Fuzhen Xuan ${ }^{3}$ \\ 1. Hubei Provincial Key Laboratory of Chemical Equipment Intensification and Intrinsic Safety, Wuhan \\ Institute of Technology, Wuhan 430205, P. R. China \\ 2. Department of Mechanical \& Aerospace Engineering, University of Strathclyde, Glasgow G1 1XJ, \\ UK \\ 3. Key Laboratory of Pressure System and Safety, MOE, School of Mechanical Engineering, East China \\ University of Science and Technology, 130 Meilong Street, PO Box 402, Shanghai 200237, PR China
}

Abstract: Bolted flange joints are indispensable components in process industries due to the good sealing, assemble and disassemble capacities. Generally, the flange rigidity characterized by the rotation angle is a key index to evaluate the sealing tightness of flange joints. However, the rotation angle of flange is usually too small (less than $1^{\circ}$ ) to monitor during the assemble and operation stages. Accordingly, a novel flexure magnifying mechanism is designed to measure the small rotation angle of flange joints under internal pressure and external bending moment. The magnification factor and calculation approach of the flexure amplification mechanism are deduced and verified by experimental data and finite element simulation. Results indicate that the proposed measuring apparatus has good performance to monitor the maximum rotation angle. It is of great interest that the measured location of the maximum rotation angle is in good agreement with that in the experiment, and the average error is $7.3 \%$, which is acceptable for practical application. Additionally, the leakage rate at the top of flange joints slowly and almost linearly increases with the increment of external bending moment ascribing to the decrease the gasket stress near the top of flange joints.

Keywords: Flexure magnifying mechanism; Flange joints; Measurement; Rotation angle; Leakage rate

\section{Introduction}

Bolted flange joints are widely utilized to connect process equipment and piping systems in

1 Author to whom correspondence should be addressed;

Electronic mail: haofeng.chen@strath.ac.uk; 
petrochemical and chemical engineering, power plant etc. from room temperature to elevated

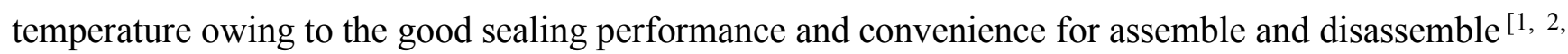
3]. However, they are also important potential leakage sources of equipment and piping systems under operating conditions, especially at high temperature. There are some reasons for the leakage behavior of bolted flange joints. First of all, the unappropriate pretightening force of bolts, such as excessive pretightening force will cause the nonuniform gasket stress along the gasket width and even the collapse behavior of gasket, while too small pretightening force will cause unadequate gasket stress. Additionally, the external bending moments produced by mechanical load and thermal gradient always aggravate the nonuniformity of gasket contact stress, which accelerates the leakage behavior of bolted flange joints. To prevent the excessive nonuniformity of gasket contact stress in practical application, the rigidity of integral flange characterized by the rotation angle must be less than $0.3^{\circ}$ under the design condition based on the ASME code ${ }^{[4]}$. However, the pretightening forces of bolts are difficult to control accurately during the assemble process, and those additional external bending moments are usually random and unpredictable. Therefore, it's necessary to monitor the very small rotation angle of flange during the assemble and operation stages to ensure the uniformity of gasket contact stress and hence the tightness of flange joints.

Precision and sensitivity are of great interest in manufacturing $[5,6,7,8,9,10]$ as well as process measuring $[11,12,13,14,15,16]$ industries, especially to improve the product quality by controlling very small geometrical parameters or errors accurately and steadily. In the field of small displacement- or deformation-controlled applications, the flexure-hinge mechanism is usually considered as an important amplifier element to enhance the measuring accuracy or processing precision ${ }^{[17,18]}$. Therefore, the rigidity and amplification, which are regarded as the key parameters of flexure-hinge mechanisms, are widely investigated by many researchers in recent years ${ }^{[19,20,21,22]}$. Recently, Tu and Xuan et al. [23, 24, 25, 26] developed various extensometers to monitor the creep strain of pipeline at elevated temperature, especially by magnifying mechanisms. However, according to the best knowledge of authors, very few references introduced the deformation monitoring of bolted flange joints during the assemble or operation process, especially the rotation angle of flange. It is necessary to investigate the measuring approach of deformation and rotation angle of flange joints, because they are main leakage resources of pressurized pipeline and equipment.

In the following work, a novel flexure magnifying mechanism is designed to measure the maximum 
rotation angle of flange joints. Accordingly, the apparatus and principle for monitoring the rotation angle of flange joints are introduced in Section 2. In Section 3, the reliability and effectiveness of the proposed flexure magnifying mechanism is verified by both experimental data and finite element simulation. The reasonable geometrical dimensions of the proposed apparatus are discussed and achieved according to those experimental data. Finally, the maximum rotation angle and its location of a pipe flange under internal pressure and external bending moment are tested and discussed.

\section{Testing apparatus and principle}

\subsection{Design of flexure magnifying mechanism}

Taking into consideration the very tiny rotation angle of flange, which is usually less than $1^{\circ}$ for integral types, and the non-uniform distribution around the circumference of flange always takes place under combined external thermo-mechanical loads, it is very hard to measure the small rotation angle of flange joints directly, as shown in Fig.1. However, when the flange joints deflect under external loads, the sealing planes of flange will generate a relative rotation. The rotation angle is a function of the relative displacement and geometrical dimension of flange joints. Therefore, it is more convenient and easier to measure the relative displacement of deformed flange planes to obtain the small rotation angle.
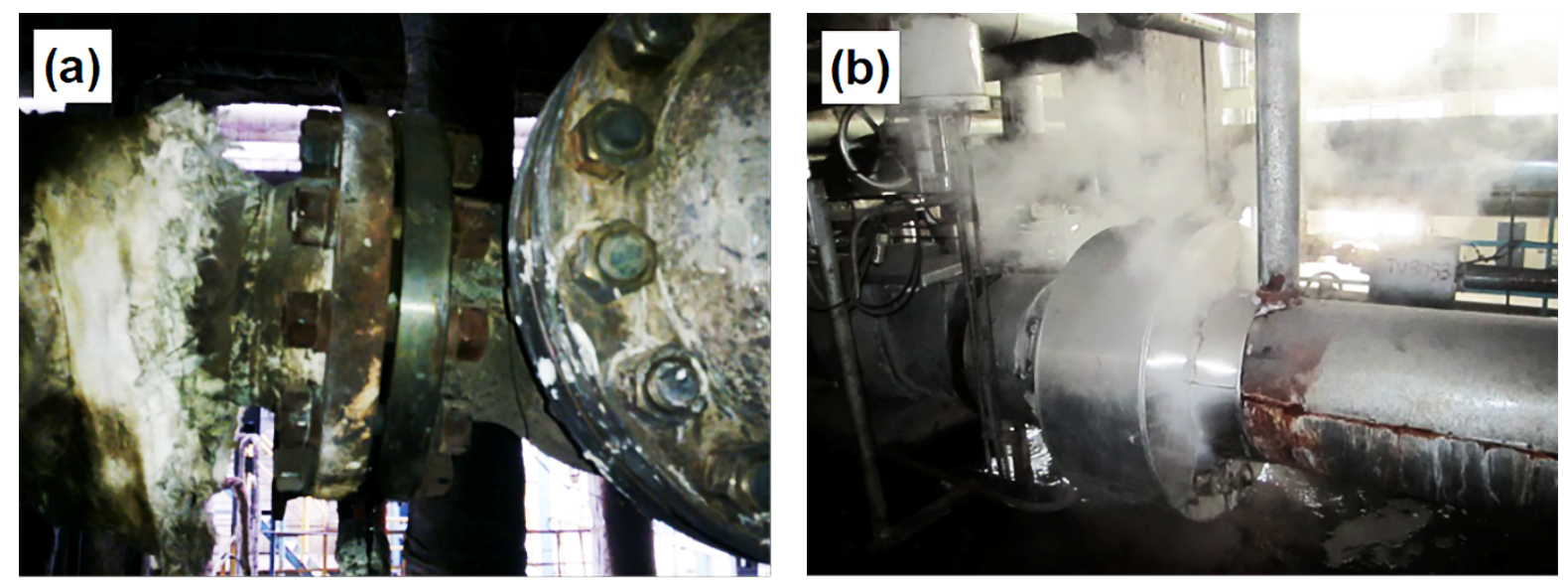

Fig. 1 High-temperature flange joints for (a) delayed coking equipment and (b) moderate-pressure superheated steam piping

The designed flexure magnifying apparatus to test the relative displacement of deformed flange joints is shown in Fig.2. It can be seen that the deflection of flange joints is measured by a three-end amplification mechanism. The extension rods $A-E$ and $A_{1}-E_{l}$ have two functions. On one hand, they can improve the magnification factor of the small displacement occurred at the points $A$ and $A_{1}$, which are fixed at the flange. On the other hand, when the flange joints are operated at elevated temperature, the 
extension rods can be used as heat insulation parts between the flange joints and the measurement elements. The main principle of the proposed flexure magnifying mechanism is to transfer the relative displacements at the specified location of flange joints to the measuring elements and then to analyze the maximum rotation angle by the proposed formula. For example, when a small deflection of the applied flange joints occurs between points $A$ and $A_{l}$, the displacements of points $A$ and $A_{l}$ are transferred to the points $C$ and $C_{l}$ by the extension rods, and then are further amplified by the rhombus mechanism at points $m$ and $n$. The distance variation between points $m$ and $n$ can be measured by a displacement sensor directly. Therefore, the true displacement between points $A$ and $A_{l}$ can be obtained by dividing the deflection between points $m$ and $n$ by the magnification factor of the measuring apparatus. Similarly, the displacements between $B$ and $B_{1}, D$ and $D_{1}$ are also available to measure the relative deflections of the other two points at flange planes. Since the deflections of flange joints in $A-C$ direction and $B-D$ direction can be tested, the maximum rotation angle of flange joints can be obtained according to the formula proposed in the following section. It is worth noting that the measuring apparatus can be utilized to measure the maximum rotation angle around the circumference of flange joints.

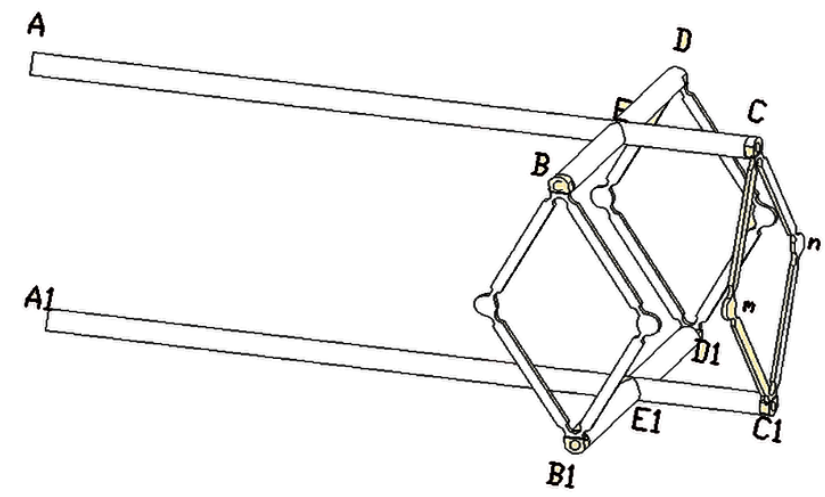

Fig. 2. Schematic diagram of measuring device

It should be noted that Fig.2 depicts a typical rhombus amplification mechanism with common hinged connections. Whereas, there are many engineering problems for common hinged structures, such as the friction force at hinged connections, lubrication, uncompact structures and so on, which may affect the measuring accuracy. In order to solve these problems, a new amplification mechanism is proposed by replacing the common hinge connection with the flexure hinge, as shown in Fig.3. Comparing with the previous common hinged connections, the flexure hinge amplifying mechanism has some obvious advantages. For example, frictionless, high precision, compact and integral mechanism and so on, which make the apparatus easier to design and process. Because the magnification factor is an important index 
to design the flexure magnifying mechanism, a reasonable magnification factor is necessary for practical engineering application.
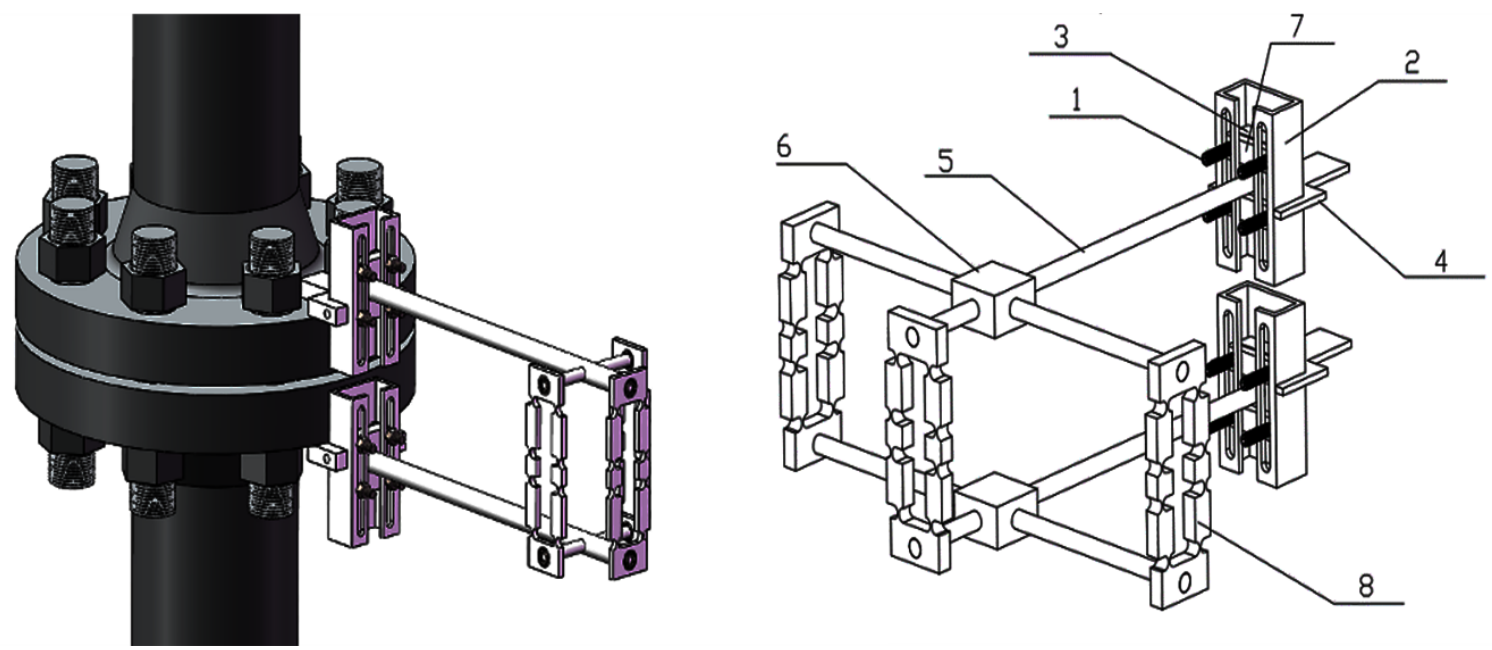

Fig. 3. Extended amplification mechanism; 1. Fastening screw, 2. Card slot, 3. Rod base, 4. Slot holder, 5. Extension bar, 6. Rod connecting block, 7. Baffle, 8. Rhombus mechanism

\subsection{Magnification factor of the measuring device}

2.2.1 Magnification factor of the measuring device with common hinge connections

The overall magnification factor of the device and geometrical dimensions of flange joints are key parameters to calculate the rotation angle based on the tested data. In which, the rhombus amplification mechanism is of more importance for the amplification behavior of the measuring device. Therefore, it is necessary to deduce the magnification factor of the rhombus mechanism with common hinge connections, as shown in Fig.2.

To obtain the magnification factor of the rhombus mechanism, we assume that the mechanical friction of the common hinge is negligible. When a given displacement is applied between points $C$ and $C_{l}$ along the $C-C_{1}$ direction, the amplified displacement is obtained based on the distance variation between points of $m$ and $n$, as depicted in Fig. 2. To describe this problem clearly, the distance of the hinge $C-n-C_{1}$ is stressed in Fig.4. The length of $C-n$ is $L$ and the angle with respect to the vertical direction is $\theta$. In this case, the magnification factor is a function with respect to the initial angle of magnifying mechanism, $\gamma$. The rod $C-n-C_{1}$ moves to $C_{3}-n_{1}-C_{2}$ under the given displacement, and then $K_{r}=\left|n n_{1}\right| /\left|C C_{3}\right|$ is the magnification factor of the rhombus amplification unit. The derivation of this magnification factor includes: 


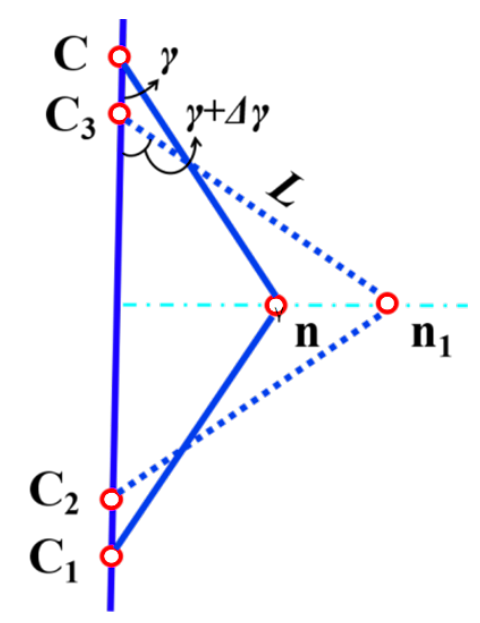

Fig. 4. Displacement amplification schematic diagram

$$
\begin{gathered}
\left|C C_{3}\right|=L(\cos \gamma-\cos (\gamma+\Delta \gamma) \\
\left|n n_{1}\right|=L \sin (\gamma+\Delta \gamma)-L \sin \gamma \\
K_{r}=\frac{\left|n n_{1}\right|}{\left|C C_{3}\right|}=\frac{\sin \gamma(\cos \Delta \gamma-1)+\sin \Delta \gamma \cos \gamma}{\cos \gamma(1-\cos \Delta \gamma)+\sin \Delta \gamma \sin \gamma}
\end{gathered}
$$

Considering that the flange deflection is very small, $\Delta \gamma$ is close to zero, then,

$$
\sin \Delta \gamma \approx \Delta \gamma, \quad \cos \Delta \gamma \approx 1-\frac{\Delta \gamma^{2}}{2}
$$

The magnification factor of the mechanism is then obtained as follows:

$$
K_{r}=\frac{-\Delta \gamma \cdot \sin \gamma+2 \cos \gamma}{\Delta \gamma \cdot \cos \gamma+2 \sin \gamma} \approx \cot \gamma
$$

In order to achieve the amplification function, $\cot \gamma$ must be greater than 1 . This means that the available range of $\gamma$ is $(0, \pi)$. Based on Eq. (5), the smaller $\gamma$ is used, the greater $K_{r}$ is obtained.

Additionally, the relative displacement of flange joints is also amplified by the extension bar, as shown in Fig.3. Therefore, the overall magnification factor of the measuring device should be the product of the magnification factor produced by the extension bar and the rhombus amplification mechanism. The magnification factor of the extension bar is the ratio of the displacement at the end of the extension rod to the original displacement at the tested point of flange, namely

$$
K_{b}=\frac{L_{b}+R_{o}}{R_{o}}
$$

In which, $L_{b}$ is the length of the extension bar, $R_{o}$ is the outer radius of flange. 
Therefore, the total magnification factor $K_{s}$ of the proposed device is

$$
K_{s}=K_{b} \cdot K_{r}=\frac{L_{b}+R_{o}}{R_{o}} \cdot \cot \gamma
$$

\subsubsection{Magnification factor of the measuring device with flexure hinge}

The flexure amplification mechanism designed is a symmetrical structure, which adopts circular flexure hinges to improve the motion sensitivity in the rotation direction. Similar to the bridge-type amplification mechanism with linear output, the proposed flexure amplification mechanism can amplify the input displacement $\Delta x$ and obtain the bidirectional output $\Delta y$, as shown in Fig. 5. The main dimensions of the flexure mechanism include the cutting radius $r$, the minimum thickness $t$, the overall thickness $w$, the hinge horizontal height difference $h$ and the hinge length $L$.

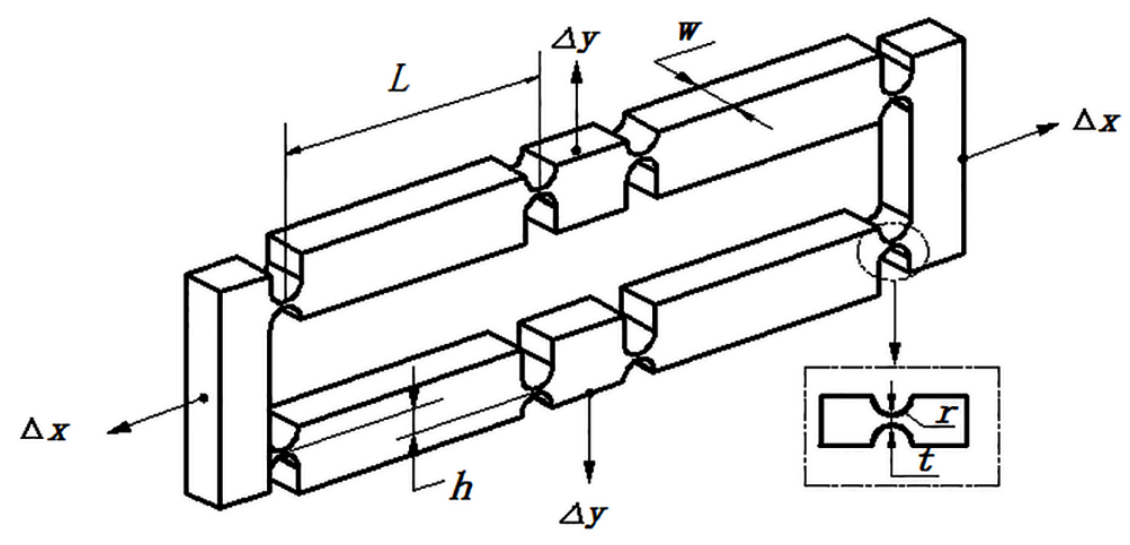

Fig. 5. Flexure hinge amplification mechanism

The hinge stiffness should be taken into account to calculate the magnification factor by introducing the flexure hinge, as shown in Fig. 6. Assume that the point $u$ is a turning pair with rotational stiffness $K_{\theta}$ and moving stiffness $K_{t}$ in the horizontal direction. To simplify the model, it is reasonable to regard the part between two hinges as a rigid connecting rod due to its greater width and shorter length. Hence, if the point $u$ shifts to $u_{l}$ and produces a small displacement $\Delta x$, the point $v$ will move to $v_{l}$ and outputs an amplified displacement $\Delta y$. 


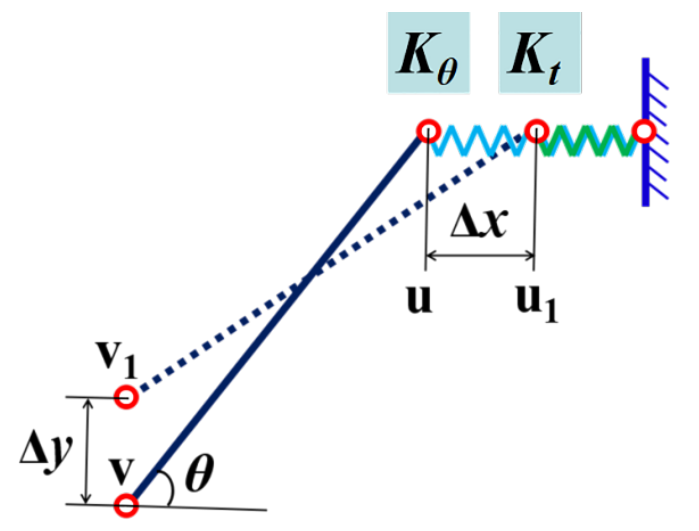

Fig. 6. Simplified stiffness model of flexure mechanism

Since the point $u$ in Fig. 6 is seemed as a rotational pair with moving rotational stiffness $K_{t}$ and the point $v$ is considered as a rotational pair with rotational stiffness $K_{\theta}$, the load analysis of the flexure mechanism can be demonstrated in Fig. 7.

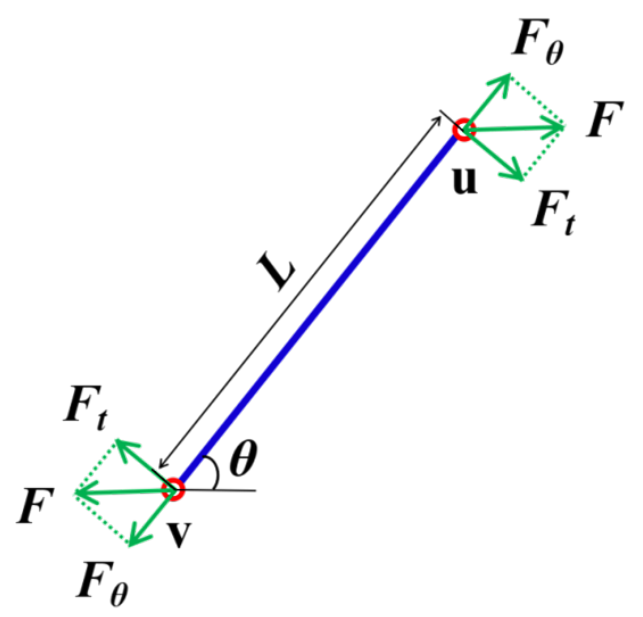

Fig. 7. Load analysis diagram of the flexure rod

Assuming that the bending moment to produce a rotation angle $\Delta \theta$ for the flexure rod $u$ - $v$ is $M_{\theta}$, the moment balance equation is:

$$
\begin{aligned}
& M_{\theta}=F \cdot L \cdot \sin \theta=K_{\theta} \cdot \Delta \theta \\
& F=K_{t} \cdot \Delta x=K_{t} \cdot \Delta L \cdot \cos \theta
\end{aligned}
$$

where, $\Delta L$ is the deformation of the flexure rod in the axial direction.

Based on the conservation of kinetic energy, we can obtain

$$
F \cdot \Delta x=F \cdot \Delta L \cdot \cos \theta+M_{\theta} \cdot \Delta \theta
$$

Substituting Eqs. (8) and (9) into Eq. (10), then

$$
F \cdot \Delta x=K_{t}(\Delta L \cdot \cos \theta)^{2}+K_{\theta} \cdot \Delta \theta^{2}
$$


Accordingly,

$$
\Delta x=\frac{K_{t}(\Delta L \cos \theta)^{2}+K_{\theta} \cdot \Delta \theta^{2}}{F}
$$

Substituting Eqs. (8), (9) into Eq. (12), it is easy to get

$$
\Delta x=\frac{K_{\theta}+K_{t} \cdot L^{2} \cdot \sin ^{2} \theta}{K_{\theta} \cdot K_{t}} \cdot F
$$

Similarly,

$$
\Delta y=\frac{L^{2} \cdot \sin \theta \cos \theta}{K_{\theta}} \cdot F
$$

Finally, the magnification factor of the flexure amplification mechanism is

$$
A=\frac{\Delta y}{\Delta x}=\frac{K_{t} \cdot L^{2} \sin \theta \cos \theta}{K_{\theta}+K_{t} \cdot L^{2} \sin ^{2} \theta}
$$

In which, the rotational stiffness $K_{\theta}$ can be described by Eq.(16) ${ }^{[27]}$, and the moving stiffness $K_{t}$ can be characterized by Eq.(17) ${ }^{[28]}$, approximately, as the following:

$$
\begin{gathered}
K_{\theta}=\frac{E}{12 w t^{3}(1.13 t+0.332)} \\
K_{t}=\frac{E w}{\left[\frac{2(2 r+t)}{\sqrt{t(4 R+t)}}\left(\arctan \sqrt{1+\frac{4 r}{t}}-\frac{\pi}{2}\right)\right]}
\end{gathered}
$$

where, $E$ is the elastic modulus of the material.

\subsection{Calculation of the rotation angle of flange joints}

The rotation angle of flange joints is usually composed by two components. The first part is induced by the pretightening of bolts, axial tension, internal pressure or thermal expansion and uniformly distributed around the hoop direction of flange. The other part is produced by the external mechanical or thermal bending and almost linearly distributed along the radial direction of flange, as demonstrated in Fig. 8. Actually, the local regions where the minimum and the maximum rotation angles take place are always considered as the most dangerous parts of flange joints. Near the maximum rotation angle area, the sealing gasket may collapse under the excessive compressive force. However, the leakage behavior is easy to occur near the minimum rotation angle region owing to the significant decrease of gasket stress. Therefore, the minimum and the maximum rotation angles and their locations of flange 
joints are critical to monitor the health status of flange joints.

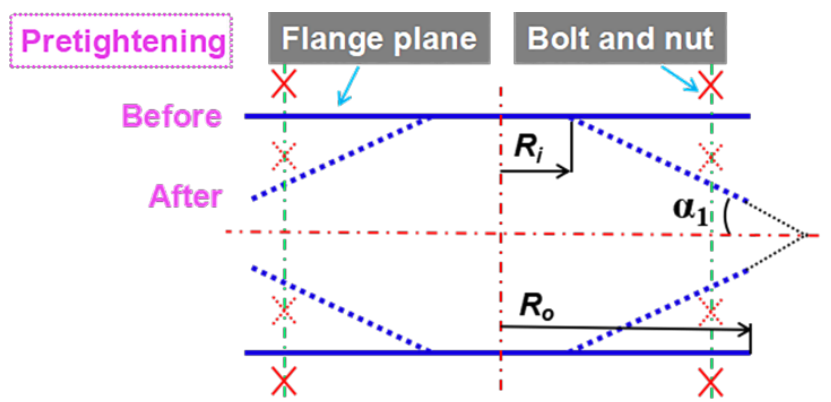

(a) Rotation angle induced by pretightening

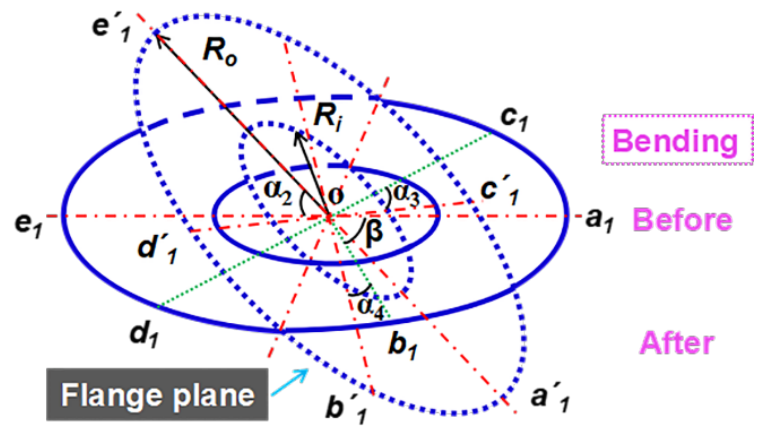

(b) Rotation angle induced by external bending

Fig. 8. Principle diagram of the maximum rotation angle of flange

Assume that $b_{1}, c_{1}$ and $d_{1}$ are the monitoring points of the flexure amplification mechanism, the minimum and the maximum rotation angles take place at the points $\mathrm{e}_{1}$ and $\mathrm{a}_{1}$, respectively, the uniform rotation angle of flange under pretightening and internal pressure is $\alpha_{1}$, the linearly distributed rotation angle of flange under external bending is $\alpha_{2}$, as shown in Fig.8. When the measured displacements of three flexure amplification mechanisms between $B-B_{l}, C-C_{l}$, and $D-D_{1}$ in Fig. 2 are $l_{b}, l_{c}$ and $l_{d}$, respectively, the rotation angle $\alpha_{1}$ can be obtained based on the symmetry of displacements at points $c_{1}$ and $d_{1}$

$$
\begin{aligned}
& \alpha_{1}=\arcsin \left(\frac{2 l_{c}-l_{b}-l_{d}}{4|E C|}\right) \\
& \alpha_{3}=\arcsin \left(\frac{\left|l_{b}-l_{d}\right|}{4|E C|}\right)
\end{aligned}
$$

If the included angle between $o-a_{1}$ and $o-b_{1}$ is $\beta$, the included angle between $o-a_{1}$ and $o-c_{1}$ is $\left(90^{\circ}-\right.$ $\beta$ ) considering that the line $o-b_{1}$ is perpendicular to the line $o-c_{1}$, as shown in Fig.8. Based on the axial displacement condition, we can obtain that 


$$
\begin{aligned}
& \tan \alpha_{3}=\cos \beta \cdot \tan \alpha_{2} \\
& \tan \alpha_{4}=\sin \beta \cdot \tan \alpha_{2}
\end{aligned}
$$

The included angle $\beta$ can be expressed as

$$
\beta=\arctan \left[\tan \alpha_{3} / \tan \alpha_{4}\right]
$$

The rotation angle under external bending moment can be expressed as:

$$
\alpha_{2}=\arctan \left[\frac{1}{2}\left(\frac{\tan \alpha_{4}}{\cos \beta}+\frac{\tan \alpha_{3}}{\sin \beta}\right)\right]
$$

It should be noted that $\alpha_{1}=\alpha_{4}$, and $\alpha_{2}=\alpha_{3}=0$ under the pretightening and internal pressure condition. If the external bending is applied, the sum of $\alpha_{1}$ and $\alpha_{4}$ is monitored by the the flexure amplification mechanism.

Based on the above equations, the total maximum and minimum rotation angles of flange joints can be obtained approximately by

$$
\alpha_{\max }=\alpha_{1}+\alpha_{2}, \quad \alpha_{\min }=\alpha_{1}-\alpha_{2}
$$

Especially, the total maximum rotation angle can be expressed as

$$
\alpha_{\max }=\arcsin \left[\frac{2 l_{c}-l_{b}-l_{d}}{2|E C|}\right]+\arctan \left[\frac{1}{2}\left(\frac{\tan \alpha_{4}}{\cos \beta}+\frac{\tan \alpha_{3}}{\sin \beta}\right)\right]
$$

Therefore, the rotation angle of flange ring under external bending moment can be obtained easily based Eqs.(18) to (25).

\section{Verification of small deformation tested by amplification mechanism}

\subsection{Verification by compression experiment}

In order to verify the reliability and accuracy of the proposed amplification mechanism to measure small deflection, the flexure amplification mechanism is designed and made by polypropylene, as shown in Fig.9. The key geometrical parameters of flexure hinge are $t=0.9 \mathrm{~mm}$ and $r=3 \mathrm{~mm}$. To discuss the effect of geometrical parameters $L$ and $w$ on the overall magnification factor, four specimens with different $L$ and $w$ listed in Table 1 are tested. 


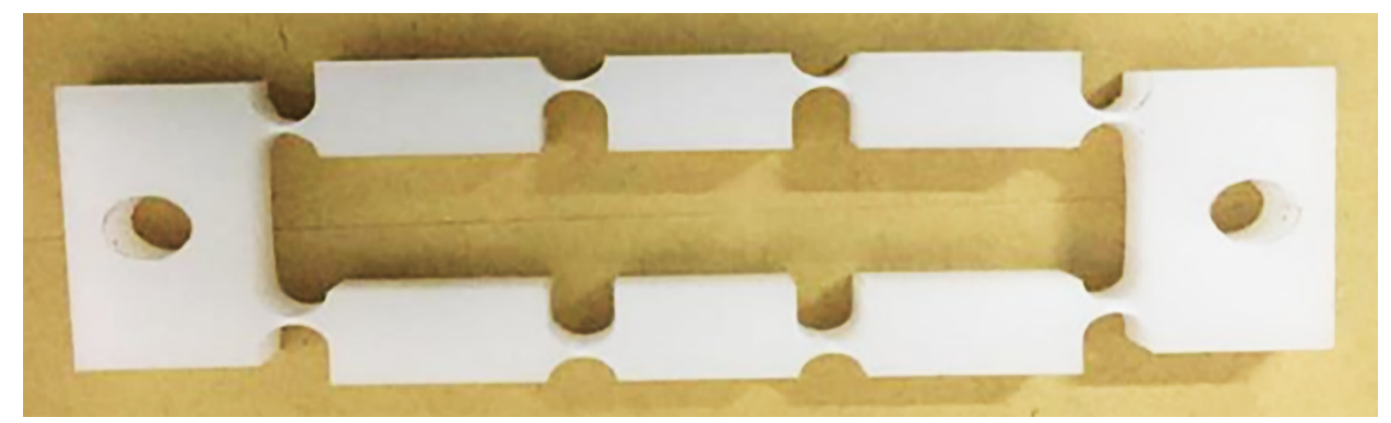

Fig. 9. Flexure amplification mechanism

Table 1 Geometrical parameters of flexure hinge

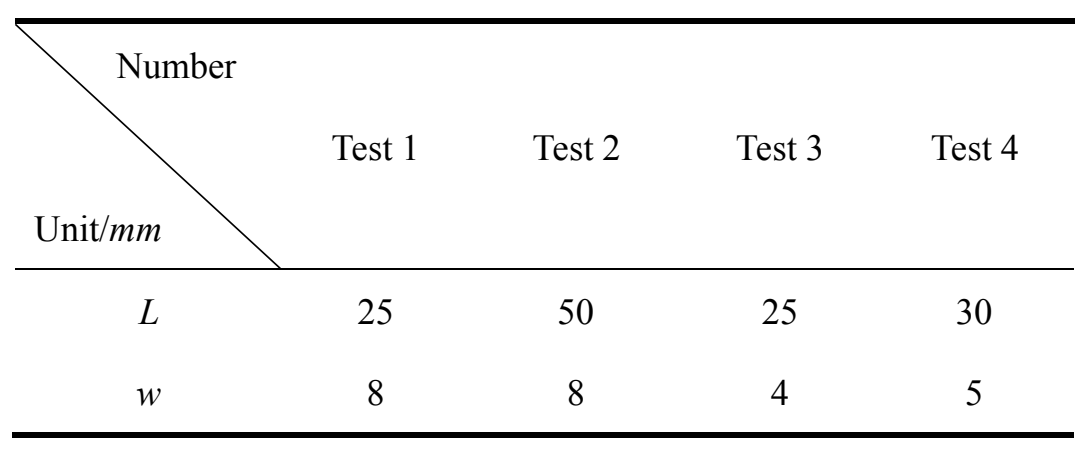

The RPL50 creep-fatigue machine is utilized to perform the experiments, as shown in Fig.10. The set-up is used to give a small displacement input with the load rate of $1 \mathrm{~mm} / \mathrm{min}$ in the vertical direction. At the same time, the displacement output of the amplifying mechanism in the lateral direction is measured by a displacement sensor.

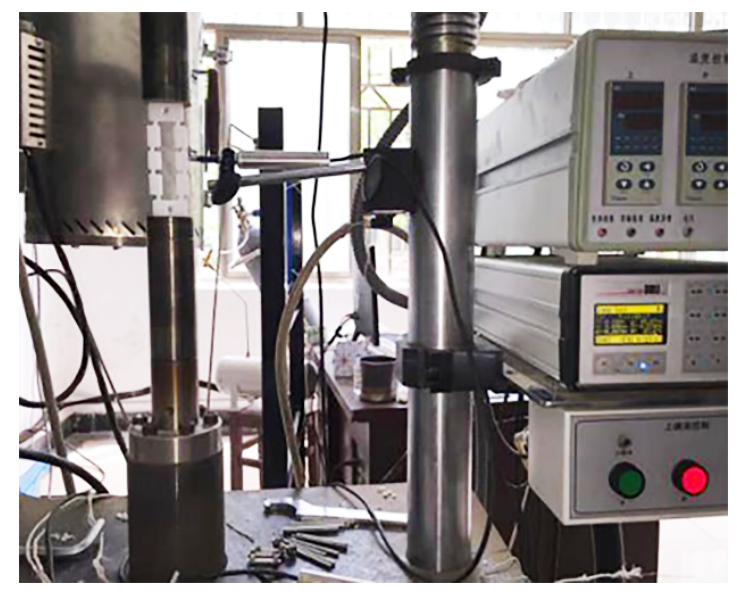

Fig. 10. Testing approach of flexure amplification mechanism 
Based on the testing specimens and procedures mentioned above, the displacement outputs from various experiments are compared with those theoretical calculations, as shown in Fig.11. Results illustrate that the analytical magnification behaviors of the flexure amplification mechanism agree very well with the experimental data, and the maximum error is less than $6 \%$. This means that the theoretical solution has relatively high accuracy to predict the magnification performance in practical engineering design. Moreover, the input and output displacements for the above four experiments are presented in Fig.12. Results clearly indicate that increasing the length $L$ or decreasing the thickness $w$ will enhance the amplification factor of the flexure mechanism.
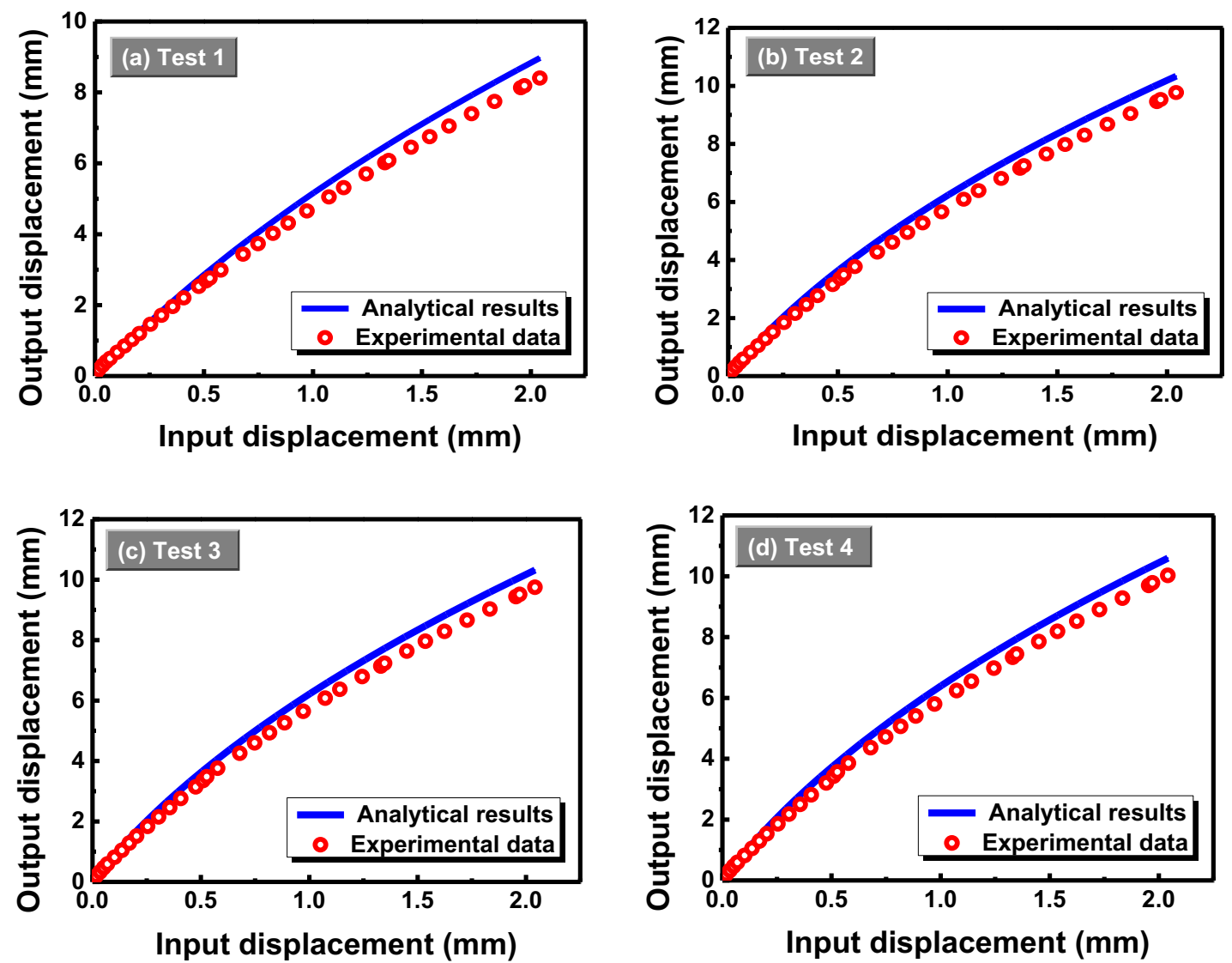

Fig. 11. Comparison between tested data and analytical results; (a) Test 1, (b) Test 2, (c) Test 3, (d) Test 4 


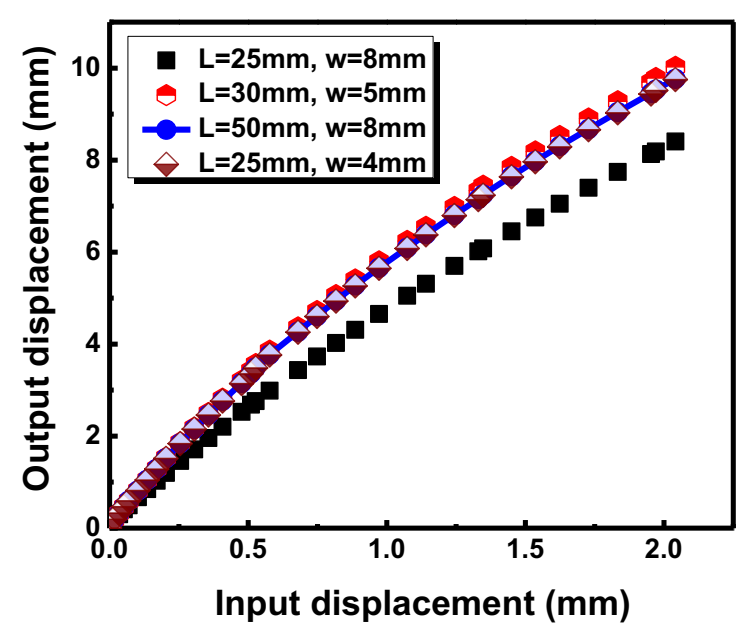

Fig. 12. Comparison of experimental results in four groups

\subsection{Validation by finite element simulation}

Considering the flexure amplification mechanism is mainly operated under the compression condition, the material properties of polypropylene under compression are tested according to the requirements of GB/T 1041-2008. The polypropylene plate is cut into square specimens with the the length of $50 \pm 0.1 \mathrm{~mm}$ and the thickness of $5 \pm 0.02 \mathrm{~mm}$. Three groups of samples are tested to promise the reliability of experimental data. Actually, the thicknesses of three specimens are $5.01 \mathrm{~mm}, 5.00 \mathrm{~mm}$, $4.98 \mathrm{~mm}$, respectively, and the samples are shown in Fig. 13.

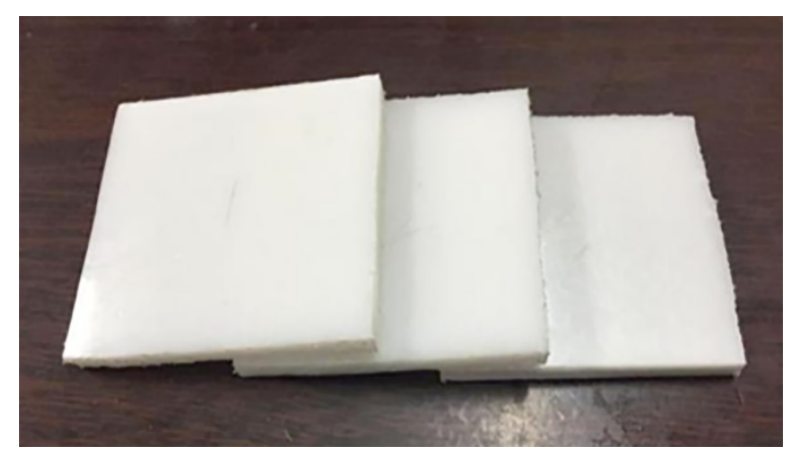

Fig.13. Polypropylene specimens

The test set-up is RPL50 creep-fatigue machine equipped with a designed compressioncompression clamp for plate-shaped specimens, as illustrate in Fig. 14. The specified compressioncompression clamp includes five components: lower and upper connecting rods, concave and convex spherical clamps and the flat clamp. It is worth noting that the concave and convex spherical clamps are designed to compensate the tiny deviation of axial compression force on the specimen surface. The axial compressive displacement is measured by a tension-compression extensometer at room temperature. The 
main compression test procedures of Polypropylene specimens include that apply the initial preload up to $10 \mathrm{~N}$, then the stress-controlled force is further used with the load rate of $12 \mathrm{KN} / \mathrm{min}$. Based on the above testing procedures, the compressive stress-strain curve is obtained, as shown in Fig. 15. Noting that the strain and stress in Fig.15 are multiplied by -1 for clear expression. Therefore, the Young's modulus of Polypropylene under compression is 1420MPa.

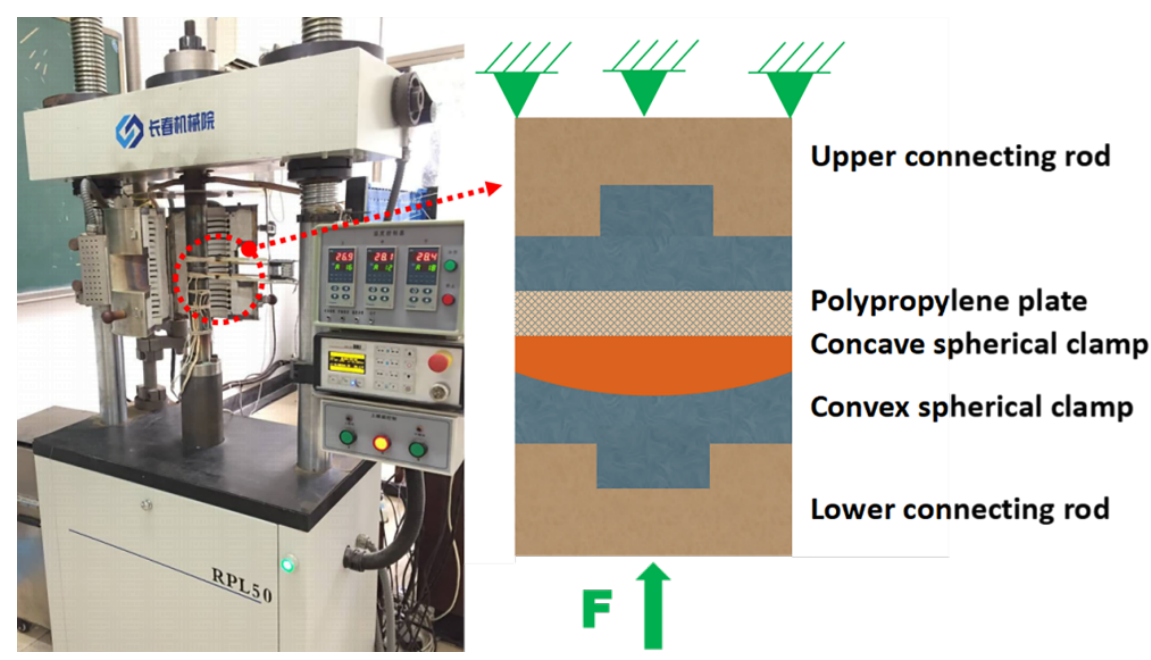

Fig. 14 Testing set-up with the designed compression-compression clamp

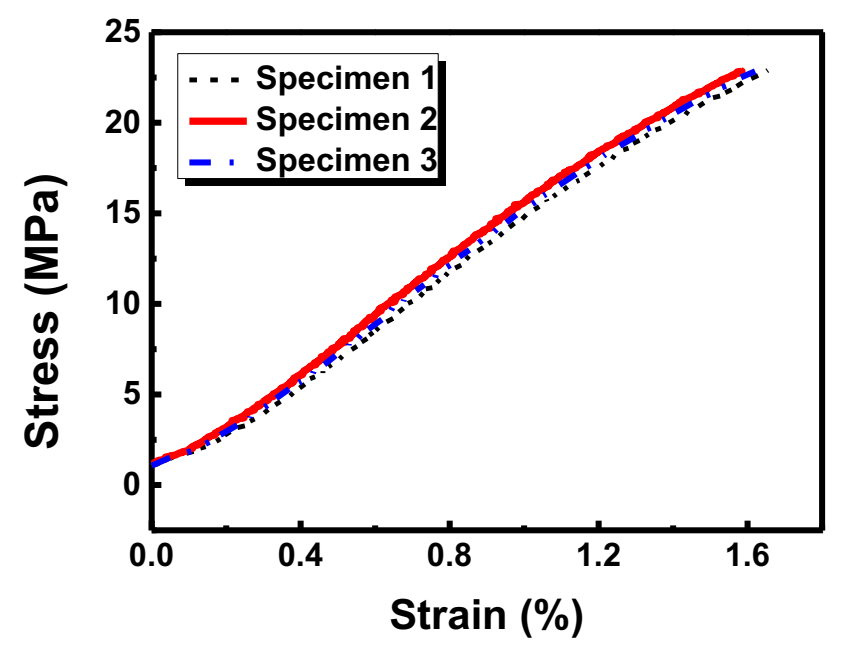

Fig. 15. Stress-strain curve of polypropylene compression test

Since the theoretical and experimental amplification are very close to each other under different geometrical parameters $L$ and $w$, the approximate stiffness equations defined according to the ratio of $t / r$ is reliable and acceptable for engineering application. Based on the above magnification performances obtained by several different specimens, the geometrical parameters of the flexure hinge are determined 
as $w=5 \mathrm{~mm}, L=30 \mathrm{~mm}$. In order to further validate the amplification behavior of the specified flexure hinge, a finite element model is established by general commercial software ANSYS to simulate its amplification performance. Taking into account the stress concentration effect near the local hinge area, the mesh density becomes obviously greater than that of the flexure rods. The overall model and the local hinge mesh are shown in Fig. 16.

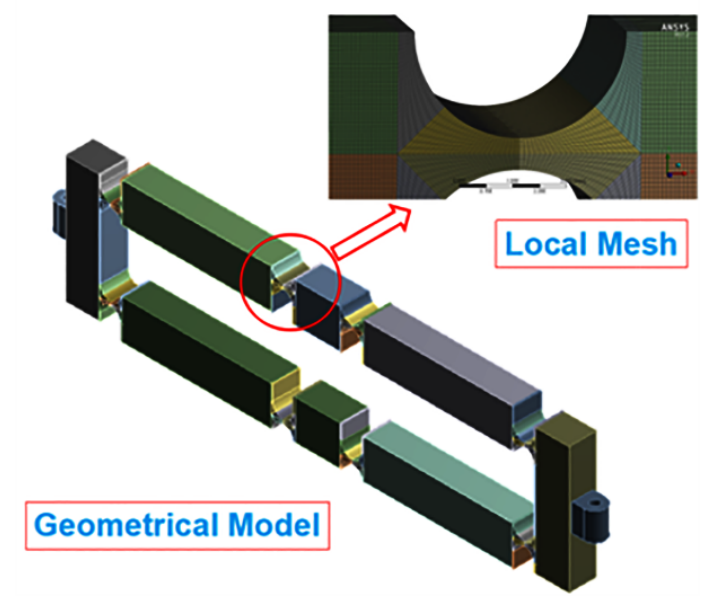

Fig.16. Finite element model of flexure hinge amplification mechanism

In order to simulate the testing condition, one end of the flexure amplification mechanism is fixed, and the other end is applied a uniformly displacement load. In order to prevent the rotation of amplification mechanism around the hinge, the degree of freedom in the normal direction at the loading end is constrained, which promises that the two end planes of the flexure amplification mechanism remain parallel to each other after deformation. The displacement contour of the flexure amplification mechanism is illustrated in Fig. 17.

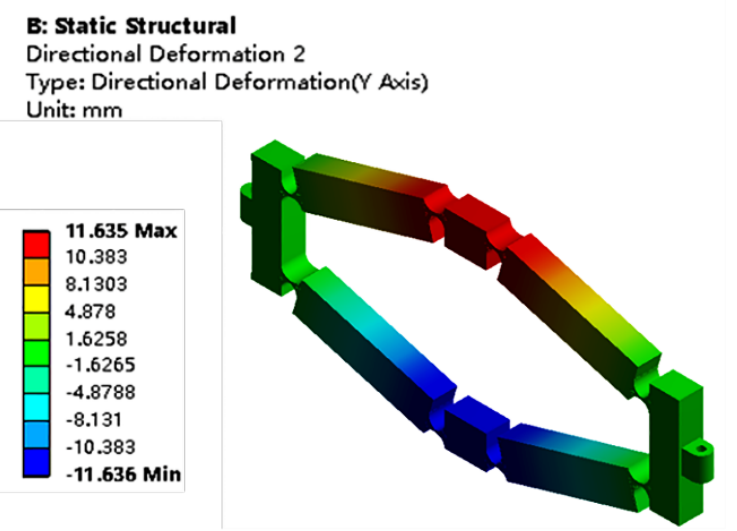

Fig.17. Displacement contour of the flexure amplification mechanism 
Fig. 18 demonstrates that the comparison of the displacement outputs obtained by finite element simulation and experiment. Results indicate that the tested amplification performance agrees well with the simulated data, and the maximum error is less than $5 \%$. This further validates the reliability of amplification mechanism with the specified flexure hinge.

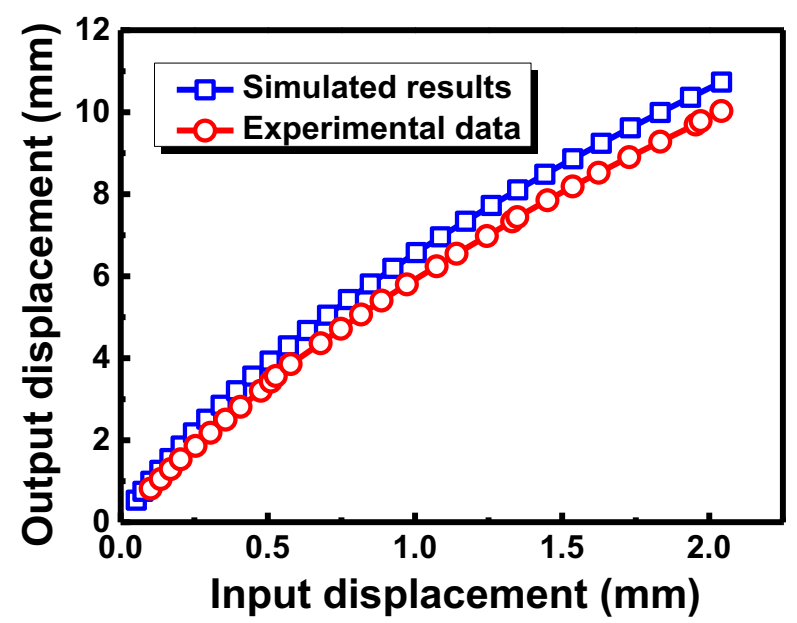

Fig.18. Comparison of finite element simulation results and experiments

\section{Measurement of rotation angles for bolted flange joints}

The pipe flange with 8 bolts, which is designed based on the HG/T 20592-2009 "Steel pipe flange" [29], is used in the following experiment. The type of the pipe flange is "HG/T 20592-2009 WN(100-63) RF S=8mm 304H", which means that the flange tested is the welding neck flange with raised face sealing, and the nominal diameter is $100 \mathrm{~mm}$, the nominal pressure is $6.3 \mathrm{MPa}$, the thickness of the pipe is $8 \mathrm{~mm}$ and the material used is $304 \mathrm{H}$. The dimension of flange is illustrated in Fig. 19(a). It is worth noting that the PTFE gasket is used in order to measure the rotation angle of flange at room temperature conveniently, and the mechanical properties of PTFE gasket are tested in detail under compression condition [30, 31, 32, ${ }^{33]}$. Moreover, the bolts are made by $35 \mathrm{CrMo}$ steel because it is one of the most widely used bolt materials in process industries ${ }^{[34,35]}$. To obtain a reasonable bolt pretightening force, the total pretightening force is imposed in a star-type tightening sequence of 1-5-3-7-2-6-4-8 and achieved in five stages, as shown in Fig. 19(b). The pretightening process is controlled by a digital torque wrench. Concretely, 20 30\% of 
the target torque for each bolt is applied initially, 50 70\% of the target torque for each bolt is imposed subsequently, after that $100 \%$ of the target torque for each bolt is used, then continue to fasten the bolt clockwisely, and finally apply $100 \%$ of the target torque until no rotation of nuts is observed. Noting that the loading sequence mentioned above is utilized for the first three pretightening stages and the maximum pretightening force for each bolt is $58.4 \mathrm{KN}$. In order to eliminate the creep effect of PTFE gasket at room temperature, the bolt pretightening force for the final stage is imposed after at least four hours when the fourth stage is finished.
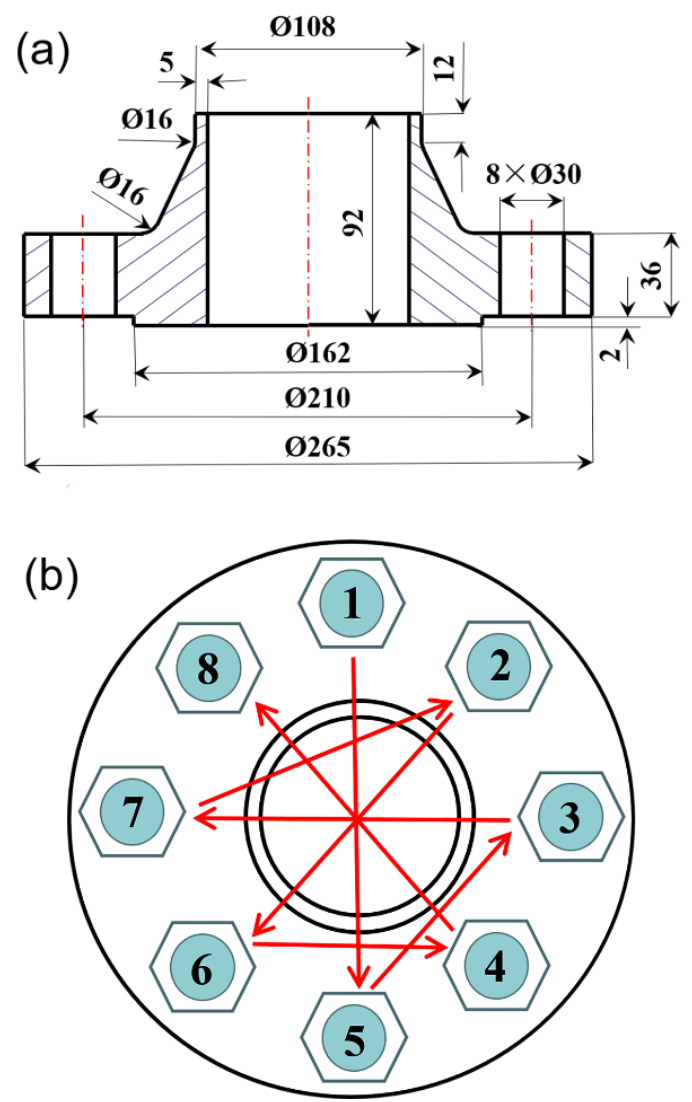

Fig.19. Schematic of flange joint; (a) Geometrical dimension of flange, (b) Pretightening sequence of bolts

The pipe is fixed on the ground by two supports. The total length of pipe is $2.5 \mathrm{~m}$, and the length between the two fixed supports is $1.5 \mathrm{~m}$. Two hydraulic-pressure controlled steel bars with the gap of $0.8 \mathrm{~m}$ are used to apply the external bending moment for pipe flange joints. Noting that the bending moment is imposed directly by two bars with equivalent and vertical upward force $F_{i}$ ( $\mathrm{i}=1$ to 6 ). The flexure amplification mechanism is installed on the flange joints after the initial pre-tightening stage is finished. It is the initial state of the flexure amplification mechanism. It is stressed that the proposed 
geometrical parameters of the flexure hinge are $w=5 \mathrm{~mm}, L=30 \mathrm{~mm}, t=0.9 \mathrm{~mm}, r=3 \mathrm{~mm}$ based on the tested data and simulation mentioned above. In this case, substituting the geometrical parameters and material properties mentioned above into Eqs. (15) to (17), the total magnification factor of the monitoring device is equal to 10.4. Considering the flange deflection is very small, the total magnification factor of the measuring device is regarded as a constant during the testing process. Additionally, the flexure amplification mechanism is installed at a random position around the circumference of flange ring. This is helpful to check that if the device can monitor the location of the maximum rotation angle. Concretely, the included angle between the extension rod and the vertical line is $130^{\circ}$ in the experiment, as illustrated in Fig.20.

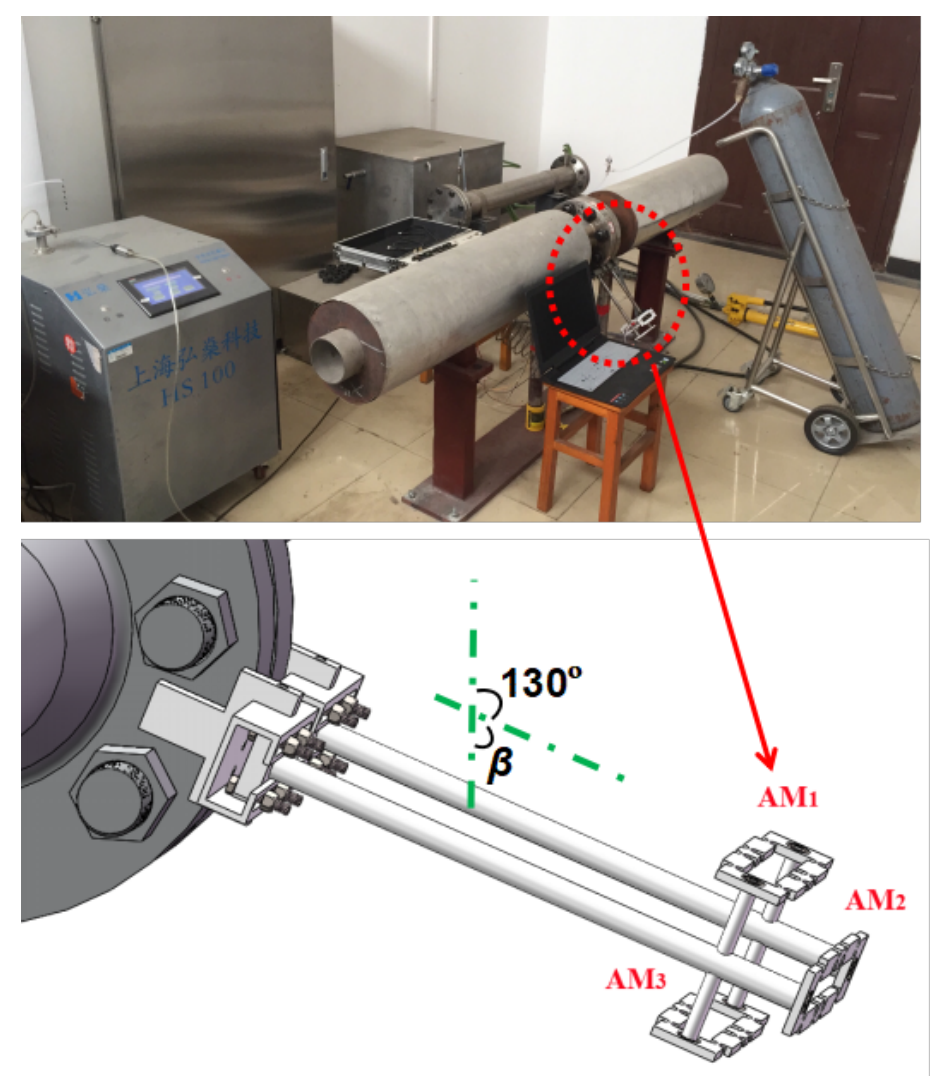

Fig.20. Experiment system of pipe flange joints with the rotation angle monitoring device

As shown in Fig. 20, the measuring device includes three flexure amplification mechanisms, $\mathrm{AM}_{1}$, $\mathrm{AM}_{2}$ and $\mathrm{AM}_{3}$ which can monitor the relative displacements at three different locations around the circumference of the flange ring. The displacement of each flexure amplification mechanism is recorded by a laser displacement sensor and checked by a vernier caliper when the external bending moment is gradually applied. Moreover, the internal pressure is applied up to $1 \mathrm{MPa}$ by a high-pressure helium gas 
tank in order to discuss the leakage rate of flange joints.

Based on the tested data from three flexure amplification mechanisms, the maximum rotation angle can be predicted based on Eqs. (18) to (23). The tested data and calculated results under various loading conditions are shown in Table 2. It should be noted that the leakage rate is tested by the helium mass spectrometer leak detector at the top of flange joints. Results present that the maximum rotation angle of flange increases with the increment of the applied force $F_{i}(\mathrm{i}=1$ to 6 ). In order to describe this phenomenon clearly, the maximum rotation angle of flange and the corresponding leakage rate are demonstrated in Fig.21. This shows that both the maximum rotation angle of flange and the tested leakage rate go up in approximately linear functions with increasing the external bending moment. Additionally, the tested included angle $\beta$ under various loading conditions is depicted in Fig.22. It is of great interest that the tested included angle $\beta$ is in good agreement with the true $\beta$ under the experiment condition, and the average error is $7.3 \%$, which is acceptable in practical engineering.

Table 2 Results of measured values and rotation angles in moment loading experiment

\begin{tabular}{|c|c|c|c|c|c|c|c|}
\hline Load case & $\begin{array}{l}\text { Force } \\
\text { (N) }\end{array}$ & $\begin{array}{l}\mathbf{A M}_{1} \\
(\mathbf{m m})\end{array}$ & $\begin{array}{l}\mathbf{A M} \mathbf{M}_{2} \\
(\mathbf{m m})\end{array}$ & $\begin{array}{l}\mathbf{A M}_{3} \\
(\mathbf{m m})\end{array}$ & $\begin{array}{l}\alpha_{4} \\
\left({ }^{\circ}\right)\end{array}$ & $\begin{array}{c}\alpha_{\max } \\
\left({ }^{\circ}\right)\end{array}$ & $\begin{array}{l}\text { Leakage rate } \\
\left(10^{-12} \mathrm{~Pa} \cdot \mathrm{m}^{3} / \mathrm{s}\right)\end{array}$ \\
\hline Initial condition & 0 & 0.00 & 0.00 & 0.00 & 0 & 0 & 0 \\
\hline Inner pressure & 0 & 4.73 & 4.82 & 4.75 & 0.057 & 0.057 & 1.5 \\
\hline $\mathrm{F}_{1}$ & 1237 & 5.27 & 5.51 & 5.44 & 0.111 & 0.154 & 2.2 \\
\hline $\mathrm{F}_{2}$ & 2474 & 5.62 & 5.95 & 5.87 & 0.147 & 0.193 & 3.1 \\
\hline $\mathrm{F}_{3}$ & 3711 & 6.36 & 6.92 & 6.83 & 0.233 & 0.313 & 4.5 \\
\hline $\mathrm{F}_{4}$ & 4948 & 7.08 & 7.85 & 7.74 & 0.315 & 0.418 & 5.0 \\
\hline $\mathrm{F}_{5}$ & 6185 & 7.30 & 8.12 & 8.01 & 0.333 & 0.444 & 5.3 \\
\hline $\mathrm{F}_{6}$ & 7422 & 7.41 & 8.26 & 8.15 & 0.344 & 0.461 & 5.9 \\
\hline
\end{tabular}




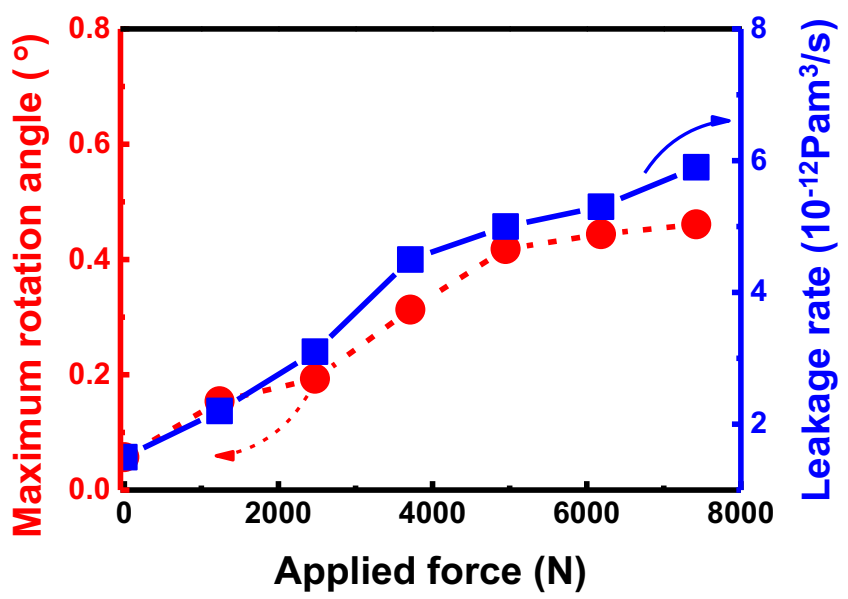

Fig.21 Effect of external bending moment on the maximum rotation angle and leakage rate of flange joints

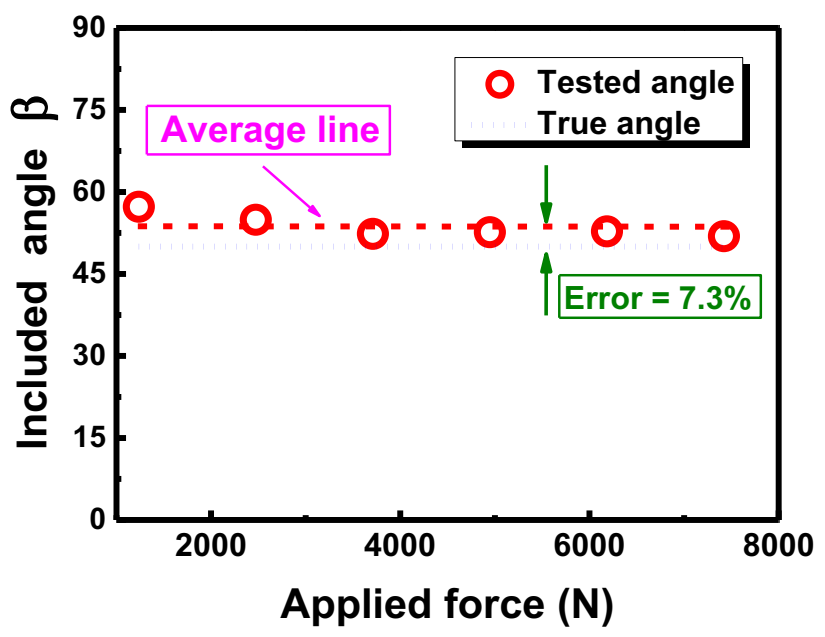

Fig. 22 Tested included angle $\beta$ under external bending moment

\section{Conclusions}

A new flexure magnifying mechanism is designed to measure the small rotation angle of flange joints. The magnification factor and the maximum rotation angle based on the proposed flexure amplification mechanism are deduced and verified by both experimental data and finite element simulation. Moreover, the rotation angle of a pipe flange joints under combined bolt pretightening force, internal pressure and external bending moment is monitored based on the apparatus. Results illustrate that the proposed flexure amplification mechanism can measure the small rotation angle of flange joints with relatively high accuracy. It is of great interest that the monitored included angle $\beta$ agrees well with the experimental data, and the average error is less than $7.3 \%$. The leakage rate at the top of the flange 
joint is measured by the Helium Mass Spectrometer Leak Detector. Results present that it increases slowly and almost linearly with increasing the external bending moment.

\section{Acknowledgments}

This work was financially supported by the National Natural Science Foundation of China (51828501 and 51305310). 


\section{References}

[1] J.Q. Guo, X.T. Zheng, Y. Zhang, H.C. Shi, W.Z. Meng, A unified continuum damage mechanics model for predicting the stress relaxation behavior of high-temperature bolting, ASME J. Press. Vessel Technol. 136 (2014): 011203.

[2] J.Q. Guo, F. Li, X.T. Zheng, H.C. Shi, W.Z. Meng, An accelerated method for creep prediction from short term stress relaxation tests. ASME J Press. Vessel Technol. 138 (2016) 031401

[3] J.Q. Guo, W.Z. Meng, X.T. Zheng, L. Tian, H.C. Shi, Prediction of stress relaxation from creep data in terms of average creep rate, J. Strain Anal. Eng. Des. 50 (2015): 15-24.

[4] American Society of Mechanical Engineers (ASME), Section VIII - Division I: Rules for Construction of Pressure Vessels, ASME Boiler \& Pressure Vessel Code (2010).

[5] X.L., Bao, J.C. Mao, X. Luo, A unified modeling and control design for precision transmission system with friction and backlash, Adv. in Mech. Eng. 8(5) (2016): 1687814016649886.

[6] X.L. Bao, J.C. Mao, Dynamic modeling method for air bearings in ultra-precision positioning stages, Adv. Mech. Eng. 8(8) (2016): 1687814016664290.

[7] J. Xu, H. Kawano, W.W. Liu, Y. Hanada, P.X. Lu, A. Miyawaki, K. Midorikawa, K. Sugioka. Controllable alignment of elongated microorganisms in 3D microspace using electrofluidic devices manufactured by hybrid femtosecond laser microfabrication, Microsyst. Nanoeng. 3 (2017): 16078.

[8] H.Y. Du, Z. Song, J.J. Wang, Z.H. Liang, Y.H. Shen, F. You. Microwave-induced shape-memory effect of silicon carbide/poly(vinyl alcohol) composite. Sensor Actuat. A-Phys. 228 (2015): 1-8.

[9] D. Zhao, W.W. Liu, S.L. Ke, Q.J. Liu. Large lateral shift in complex dielectric multilayers with nearly parity-time symmetry. Opt. Quant. Electron. 50(8): 323.

[10] S.L. Ke, J.X. Liu, Q.J. Liu, D. Zhao, W.W. Liu. Strong absorption near exceptional points in plasmonic waveguide arrays. Opt. Quant. Electron. 50(8): 318.

[11] M. Hou, Y. Wang, S. Liu, J. Guo, Z. Li, P.X. Lu, Sensitivity-enhanced pressure sensor with hollowcore photonic crystal fiber, J. Lightwave Technol. 32(23) (2014): 4035-4039.

[12] M. Hou, Y. Wang, S. Liu, Z. Li, P.X. Lu, Multi-components interferometer based on partially filled dual-core photonic crystal fiber for temperature and strain sensing, IEEE Sensors J. 16(16) (2016): 61926196.

[13] J. Shao, S.H. Liu, Photonic generation of filter-free ultrawideband monocycle and doublet signal using single semiconductor optical amplifier in counter-propagation scheme, Optical Engineering 55(2) (2016): 026117.

[14] X. Wei, L. Zhou, S.T. Liu, Development of gold-doped carbon foams as a sensitive electrochemical sensor for simultaneous determination of $\mathrm{Pb}$ (II) and Cu (II), Chem. Eng. J. 284 (2016): 650-656. 
[15]Y. Qin, L. Cheng, X. Zhou, Multi-Sensor Tracking of a Maneuvering Target Using Multiple-Model Bernoulli Filter. IEICE Trans. Fund. Electron. Comm. Comput. Sci. 98(12) ( 2015): 2633-2641.

[16]Y. Sun, D.M. Liu, P. Lu, Q.Z. Sun, W. Yang, S. Wang, L. Liu, J.S. Zhang. Dual-Parameters Optical Fiber Sensor with Enhanced Resolution Using Twisted MMF Based on SMS Structure. IEEE Sens. J. 17(10) (2017): 3045-3051.

[17] L. Clark, B. Shirinzadeh, J. Pinskier, Y. Tian, D. Zhang, Topology optimisation of bridge input structures with maximal amplification for design of flexure mechanisms, Mech. Mach. Theory 122 (2018): 113-131.

[18] S. Šalinić, A. Nikolić, A new pseudo-rigid-body model approach for modeling the quasi-static response of planar flexure-hinge mechanisms, Mech. Mach. Theory 124 (2018): 150-161.

[19] N. Lobontiu, Compliance-based matrix method for modeling the quasi-static response of planar serial flexure-hinge mechanisms, Prec. Eng. 38.3 (2014): 639-650.

[20] Y. Ni, Z. Deng, X. Wu, J. Li, L. Li, Modeling and analysis of an over-constrained flexure-based compliant mechanism, Measurement 50 (2014): 270-278.

[21] M. Jouaneh, R. Yang, Modeling of flexure-hinge type lever mechanisms, Prec. Eng. 27(4) (2003): 407-418

[22] U. Bhagat, B. Shirinzadeh, L. Clark, P. Chea, Y. Qin, Y. Tian, D. Zhang, Design and analysis of a novel flexure-based 3-DOF mechanism, Mech. Mach. Theory 74 (2014): 173-187.

[23] H.C. Zhang, J.H. Jia, N. Wang, X.Y. Hu, S.T. Tu, S.P. Zhou, Z.D. Wang, Development of on-line monitoring systems for high temperature components in power plants. Sensors 13(2013): 15504-15512. [24] J.H. Jia, X.Y. Hu, Z.L. An, F.Z. Xuan, S.T Tu, Design and verification of a sensing device for deformation measurement of high temperature pipes, J. Press. Vessel Technol. 133(2011):041601.

[25] J.H. Jia, X.Y. Hu, N. Wang, S.T. Tu, Test verification of an extensometer for deformation measurement of high temperature straight pipes, Measurement 45(2012):1933-1936.

[26] Hu X.Y., Jia J.H., Wang N., Xia X.M., Wang Z.D., Tu S.T. Design and test of an extensometer for strain monitoring of high temperature pipelines. J. Press. Vessel Technol. 134(2012):044501.

[27] S. Smith, D. Chetwynd, Bowen D, Design and assessment of monolithic high precision translation mechanisms, J. Phys. E 20(1987): 977-983.

[28] N. Lobontiu. Compliant mechanisms: design of flexure hinges. CRC Press; 2003.

[29] Chemical Industry Standards of the People's Republic of China, HG/T 20592-2009 "Steel pipe flange", Issued by Ministry of Industry and Information Technology of the People's Republic of China (2009).

[30] X.T. Zheng, X. Wen, W. Wang, J.Y. Gao, W. Lin, L.W. Ma, J.Y. Yu, Creep-ratcheting behavior of PTFE gaskets under various temperatures, Polym Test. 60(2017): 229-235. 
[31] X.T. Zheng, X. Wen, J.Y. Gao, J.Y. Yu, W. Lin, J.M. Xu, Temperature-dependent ratcheting of PTFE gaskets under cyclic compressive loads with small stress amplitude. Polym Test. 57(2017): 296-301.

[32] X.T. Zheng, X.H. Zhang, L.W. Ma, W. Lin, J.Y. Yu, J.M. Xu, Rate-dependent mechanical characteristics of polytetrafluoroethylene (PTFE) gaskets under cyclic pulsating compression. Polym Bull. 1(2018): 1-14.

[33] X.T. Zheng, H.Y. Wang, W. Wang, W. Lin, L.W. Ma, J.M. Xu, J.Y. Yu, Compressive ratcheting effect of expanded PTFE considering multiple load paths, Polym Test. 61(2017):93-99.

[34] X.T. Zheng, K.W. Wu, W. Wang, J.Y. Yu, Low cycle fatigue and ratcheting behavior of 35CrMo structural steel at elevated temperature, Nucl. Eng. Des. 314(2017): 285-292.

[35] W. Wang, X.T. Zheng, J.Y. Yu, W. Lin, C.G. Wang, J.M. Xu, Time-dependent ratcheting of 35CrMo structural steel at elevated temperature considering stress rates, Mater. High. Temp. 34(2016):172-178. 